Acta Crystallographica Section A

Foundations of Crystallography

ISSN 0108-7673

\section{Quantum Crystallography: Electron Density and Bonding, a Microsymposium}

\author{
Seppo Manninen
}

Department of Physical Sciences, University of Helsinki, Finland

The XIX Congress and General Assembly of the International Union of Crystallography took place 6-15 August 2002 in Geneva, Switzerland. The scientific program was divided into Keynote Lectures, Microsymposia and Poster Presentations. One of the 95 microsymposia was entitled Quantum Crystallography: Electron Density and Bonding. It was chaired by Genevieve Loupias, University of Paris VI and Seppo Manninen, University of Helsinki.

The program of the microsymposium consisted of five invited lectures. The topic was approached from different starting points and by various methods. Two lectures concentrated on traditional quantum crystallography: the application of quantum-mechanical methods to understand the structural properties and related electron densities and bonding features of large molecular systems. In the other three lectures, the problem was tackled somewhat differently: two alternative techniques, both based on the determination of the electron momentum densities, were presented. High-resolution Compton scattering gives direct information on the ground-state electronic properties. Third-generation synchrotron facilities have made it possible to use this technique to obtain detailed information about the electron-electron correlation effects and bonding features in simple systems. Another interesting approach is to apply electron coincidence spectroscopy, where the scattered electron is measured in coincidence with the ejected electron, so-called $(e, 2 e)$ spectroscopy. A nice feature of this method is that instead of a one-dimensional projection of the momentum density, obtained in a conventional Compton scattering experiment, the full collision kinematics is determined. A disadvantage in the case of solid targets is multiple electron scattering and a requirement of thin samples due to the strong interaction and absorption of electrons. In the last talk, the possibility of using experimental X-ray data to extract constrained Hartree-Fock wavefunctions was discussed. Based on these wavefunctions, one is able to calculate electron localization information and analyse the charge density.

Two articles in this issue describe in more detail some of the topics presented in the microsymposium. Erich Weigold, one of the pioneers in $(e, 2 e)$ spectroscopy, and his co-workers demonstrate the potential of electron spectroscopy in momentum density studies of solids. Dylan Jayatilaka \& Daniel Grimwood utilize $\mathrm{X}$-ray diffraction data on molecular crystals and discuss in detail information that can be obtained in the position space from the derived electron localization functions. 